\title{
SYNTHESIS OF CARBON NANOSTRUCTURES IN A PLASMA JET REACTOR
}

\author{
Ravil' H. Amirov, Irina N. Atamanyuk, Alexander S. Tyuftyaev, \\ Marina B. Shavelkina, Evgeny I. Shkol'nikov \\ Joint Institute for High Temperatures of Russian Academy of Sciences, http://www.jiht.ru \\ 125412 Moscow, Russian Federation \\ mshavelkina@gmail.com
}

Abstract. In this paper we propose a simple approach to produce nanocrystalline carbon materials with a narrow pore size distribution: the evaporation and condensation by a plasma jet. Synthesized samples were characterized by physic chemical methods. It demonstrated the possibility to application of plasma jet reactor to synthesis of nanostructured materials of a predetermined particle size and a pore structure in large quantities.

Keywords: catalysis, mesoporous structure, decomposition of hydrocarbons, plasma torch, synthesis, carbon nanomaterials, graphene, nanotubes

PACS: 52.50.Nr, 81.07.-b

Bibliography - 4 references

Received 1.10.15

RENSIT, 2015, 7(2):175-179

DOI: $10.17725 /$ rensit.2015.07.175

CONTENTS

1. INTRODUCTION (175)

2. Materials and Methods (175)

3. Results and Discussion (176)

4. ConClusion (178)

REFERENCES (178)

\section{INTRODUCTION}

One of the priorities in the development of new functional materials with desired properties is to obtain a bulk carbon nanomaterials based on fullerenes (fullerites), carbon nanotubes (CNTs), graphene flakes and others. This is due to the properties of these materials such as superconductivity, field emission, no wetting surface, ultra-high specific surface in combination with the electrical conductivity [1]. Some possibilities of use of these materials are to build supercapacitor or electrodes in energy conversion devices [2-3], selective catalysts and sorbents.

The main technical problem, hampering the use of three-dimensional form of carbon that are based on only covalent bonds, is the lack of good synthesis methods with reproducing results and that are scalable. Existing methods of producing fullerenes, nanotubes, et al., that are based on the use of electric arc and laser evaporation, have a number of disadvantages associated with the low efficiency of the process. Low-temperature method for producing carbon nanomaterials - a method of chemical vapor deposition, based on the decomposition of hydrocarbons in the presence of catalysts, requires careful manufacture of substrates with nanostructured catalyst. A widely used method of magnetron sputtering in a vacuum is also characterized by low productivity.

Very promising method in this respect is the use of the plasma torch [4]. The advantages of this method compared with the most common electric arc method are: the absence of restrictions on the injected power and the consequent possibility of a significant increase in productivity; ability to work with initial materials in various physical states (powders, gases and liquids); possible optimization of the process by an independent pressure control, energy input and expense ratio of the plasma gas, starting material and a catalyst; possibility of forming the stream using various auxiliary devices (nozzles), and by changing the geometrical parameters.

\section{MATERIALS AND METHODS}

In the proposed approach the initial carbonaceous materials for the synthesis of graphene materials (in the form of hydrocarbon gas), together with a working gas (argon, helium) are fed into the plasma torch DC power up to $40 \mathrm{~kW}$, whereupon their evaporation occurs in the plasma jet with further 
quenching formed carbon vapor as solid carbon with the precipitation in the gas volume, and the metal surface. The original design solution for the plasma torch - widening the channel and vortex stabilization of the plasma jet provides dimensional stability, high evaporation rate and productivity.

The main parameters were varying an atmosphere pressure (150-730 Torr) and hydrocarbon flow rate for 10-20 min experiment. For each experiment, the input of hydrocarbons was carried out after the establishment of the temperature field in a graphite reactor (determined by the temperature of water in the cooling circuit) and leveling the electrical characteristics of the plasma torch. Technical gases were used as the source for raw hydrocarbons: methane, acetylene, and the mixture - propane butane in the ratio $30: 70 \%$. The flow rate of hydrocarbons was varied between 0.2 to $6.3 \mathrm{~g} / \mathrm{s}$.

The current value of the plasma torch is maintained constant in all the experiments and is equal to $350 \mathrm{~A}$ and $400 \mathrm{~A}$ for argon and helium, respectively. A typical arc voltage varied between 50 and $110 \mathrm{~V}$, depending on the experimental conditions (atmosphere pressure and flow rate). Plasma-forming gases - helium, and argon were used with a flow rate varying from 0.5 to $3.8 \mathrm{~g} / \mathrm{s}$.

The product was characterized by electron microscopy (SEM MIRA 3 TESCAN with Schottky field emission cathode in a high-vacuum) and Raman scattering (Ntegra Spectra, the excitation wavelength was $532 \mathrm{~nm}$, emission power was adjusted so as to prevent overheating of the material). To determine the characteristics of the porous structure (pore volume, pore radius, surface area) used a relatively new method of adsorption "limited evaporation" (LE), based on the analysis of the kinetics of vaporization of the adsorbate from the test material, and classical BET method using low-temperature nitrogen adsorption - for evaluating the specific surface of materials. The effectiveness of the synthesis, thermal stability and phase composition of the carbon products were evaluated by the thermal analysis method on synchronized thermoanalyser STA 409PC Luxx (NETZSCH) during linear heating of the hitch of the sample on air with the speed of $10 \mathrm{~K} / \mathrm{min}$ in the temperature interval from room temperature to $1000^{\circ} \mathrm{C}$.

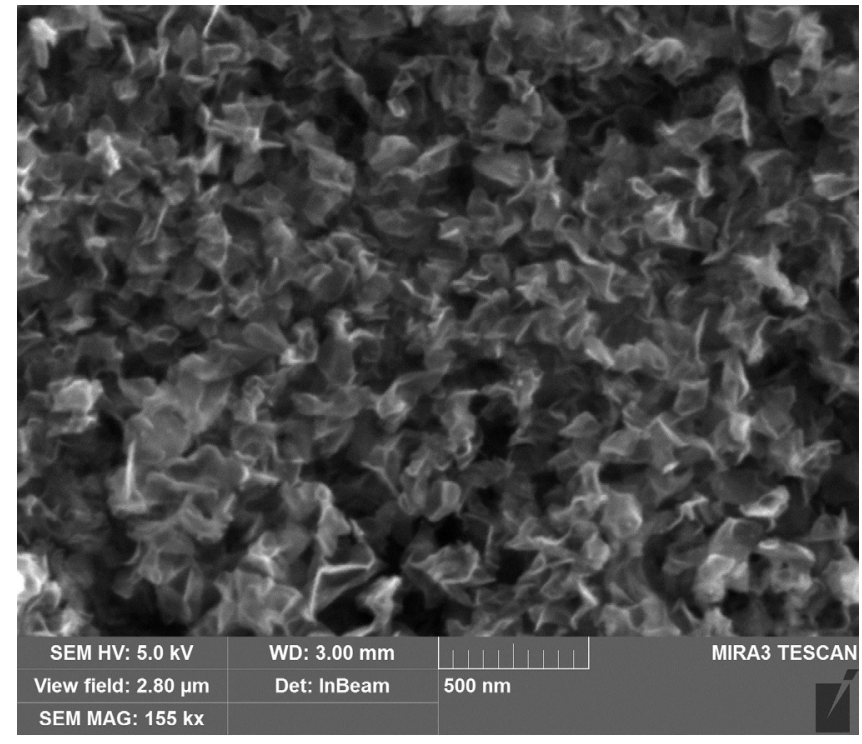

Fig. 1. Morphology of the plasma jet pyrolysis products propanebutane in the helium atmosphere 710 Torr, $400 \mathrm{~A}$, at a flow rate of the hydrocarbon $0.916 \mathrm{~m}^{3} / \mathrm{h}$ and of helium $0.75 \mathrm{~g} / \mathrm{s}$.

\section{RESULTS AND DISCUSSION}

By pyrolysis in thermal plasma basic types of carbon structures: amorphous carbon, graphite and nanotubes with different numbers of layers and different morphologies, and graphitized carbon particles were received.

Experiments on the pyrolysis of propanebutane mixture showed that the highest yield of graphene materials are obtained under the following conditions: atmosphere - helium, pressure -710 Torr consumption of hydrocarbon $-0.294 \mathrm{~m}^{3} / \mathrm{h}$. The quantity of amorphous carbon was $4 \mathrm{wt.} \%$, and the graphitized particles - less than 2 wt.\% (Fig. 1).

Depending on the type of plasma gas, pressure and the rate of its flow and the flow rate of propanebutane mixture morphology of product synthesis varies from crumpled structure (Fig. 1), obtained in 350-710 Torr argon flow 3.5-3.75 g/s and 710 Torr helium with flow 0.75 and 350 Torr $-0.95 \mathrm{~g} / \mathrm{s}$ up to large flakes with a transverse dimension $600 \mathrm{~nm}$ in 350-710 Torr helium at a flow rate of $0.75 \mathrm{~g} / \mathrm{s}$.

Depending on the type of atmosphere gas, pressure and the rate of flow and the flow rate of propane-butane mixture the synthesized product morphology is varied from crumpled structure (Fig. 1), obtained in argon atmosphere at 350-710 Torr, flow rate $3.5-3.75 \mathrm{~g} / \mathrm{s}$ and in helium atmosphere at 710 Torr with helium flow rate of $0.75 \mathrm{~g} / \mathrm{s}$, in helium atmosphere at 350 Torr with helium flow rate of $0.95 \mathrm{~g} / \mathrm{s}$ up to large flakes with a transverse dimension $600 \mathrm{~nm}$ synthesed in helium atmosphere $350-710$ Torr at a helium flow rate of $0.75 \mathrm{~g} / \mathrm{s}$. 


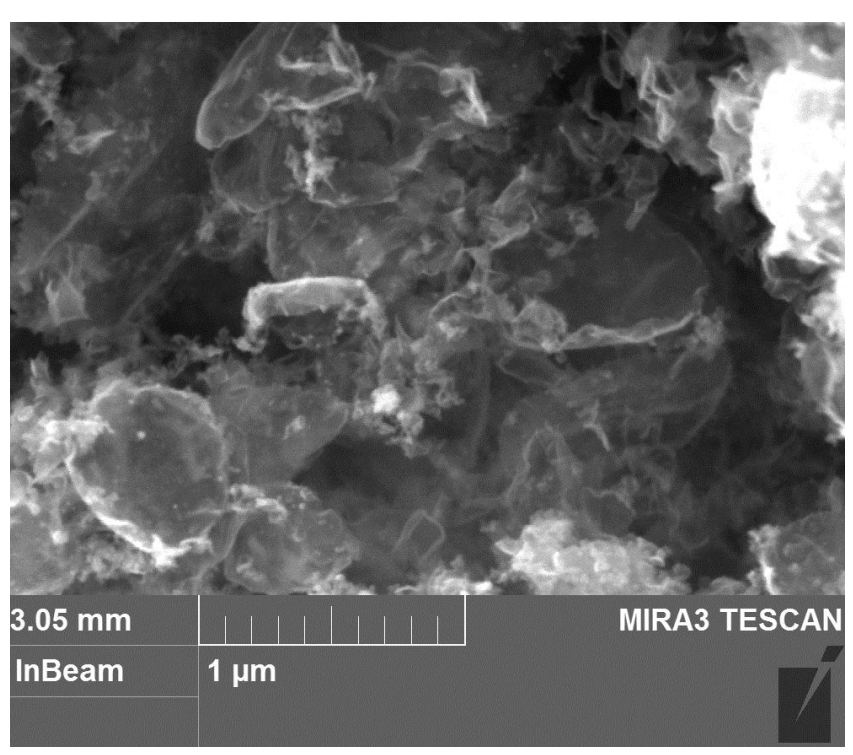

Fig. 2. Morphology of product plasma jet methane pyrolysis in the atmosphere of helium 500 Torr, $400 \mathrm{~A}$, at a rate of helium 0.75 $\mathrm{g} / \mathrm{s}$ and $a$ bydrocarbon $0.95 \mathrm{~m}^{3} / \mathrm{h}$.

Optimal conditions for the synthesis of graphene materials produced by pyrolysis of methane differ mainly by higher flow rate of carbon source $(0.77$ $1.42 \mathrm{~m}^{3} / \mathrm{h}$ ). In the experiments we used methane of two marks: mark $\mathrm{A}$ and technical. According to electron micrographs and thermogravimetry of synthesis products, from pure methane in the helium atmosphere at 350 Torr, with flow rate of $0.75 \mathrm{~g} / \mathrm{s} 65$ wt. $\%$ of graphene structures were formed, oxidated in a characteristic range for the oxidation of carbon nanotubes. The transverse size of the graphene material is 400-600 $\mathrm{nm}$. In an argon atmosphere at 350 Torr, with a flow rate of $3.5 \mathrm{~g} / \mathrm{s}$, a current of 350 A the graphene structures with carbon nanotubes are formed and there is an increase in the number of (up to 11 wt.\%) of an amorphous carbon and up to 17 wt. $\%$ - of the graphitized particles (Fig. 2).

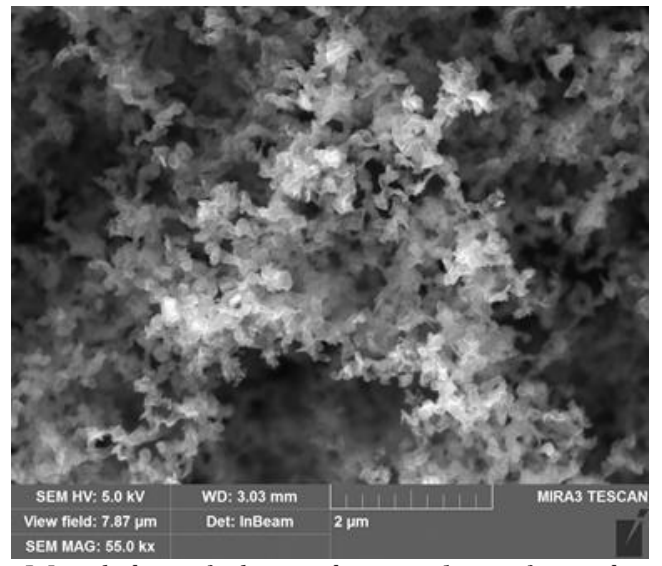

Fig. 3. Morphology of the pyrolysis products of acetylene in 150 Torr helium atmosphere, $400 \mathrm{~A}$, at a rate of bydrocarbon 0.63 $\mathrm{m}^{3} / \mathrm{h}$ and of belium $0.75 \mathrm{~g} / \mathrm{s}$.

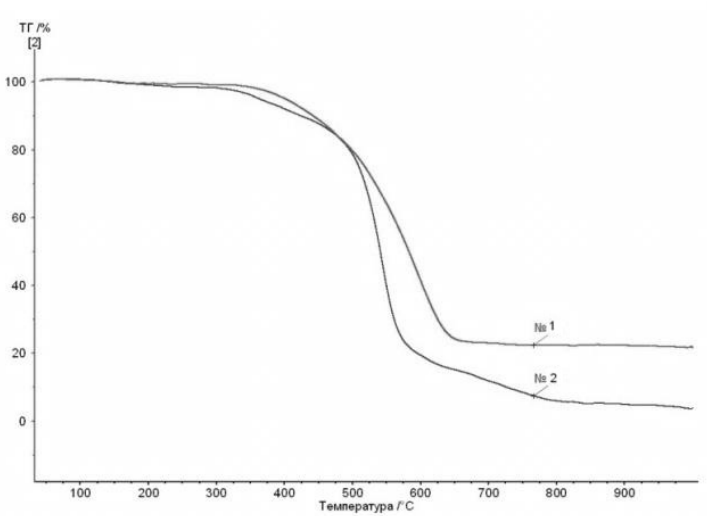

Fig. 4. Thermogravimetry of plasma jet synthesis products by pyrolysis of methane (1) and of propane-butane (2) in a helium atmosphere 500 Torr, $400 \mathrm{~A}$.

Upon decomposition of acetylene in a plasma of argon and helium at pressures 150-350 Torr a crumpled graphene structures are obtained (Fig. 3). Flow rate of acetylene was $0.2-0.63 \mathrm{~m}^{3} / \mathrm{h}$. In argon atmosphere at a flow rate of $3.6 \mathrm{~g} / \mathrm{s}$, more than 90 wt $\%$ of carbon fibers and nanofibers are formed.

According to thermogravimetric analysis (Fig. 4) thermal stability of nanostructures obtained by pyrolysis methane and by propane-butane mixture is not significantly different from each other.

Specific surface for the samples with a transverse dimension of $300-450 \mathrm{~nm}$ does not exceed $270 \mathrm{~m}^{2} / \mathrm{g}$ according to BET. Studies of surface properties were also carried out by limited evaporation (LE) method, based on the analysis of the kinetics of vaporization of the adsorbate from the test material. Fig. 5 shows the basic parameters of the porous structure of the samples obtained by LE using benzene as an adsorbate. The surface of the crumpled graphene materials obtained in 710 Torr of helium is more than the surface of the flakes synthesized at 350 Torr helium at the same flow rate of hydrocarbon $-0.294 \mathrm{~m}^{3} / \mathrm{h}$. The angle

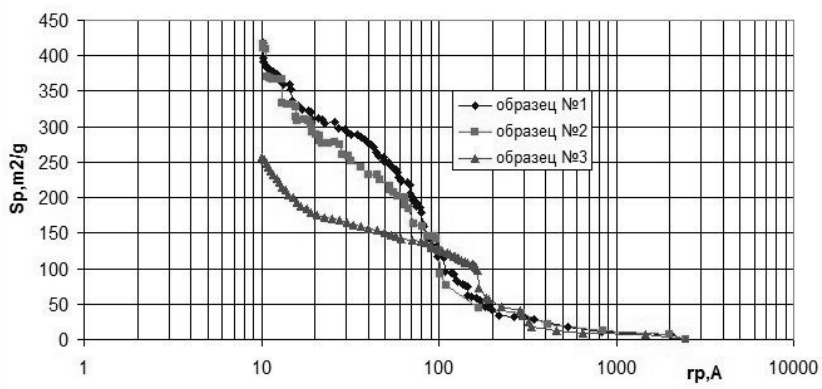

Fig. 5. Cumulative distribution curves of specific surface in depending of pore radius. The specific surface area of samples № 1 (source-propane-butane) and № 2 (methane) is about $400 \mathrm{~m}^{2} / \mathrm{g}$, sample №3 (acetylene) - $250 \mathrm{~m}^{2} / \mathrm{g}$. 


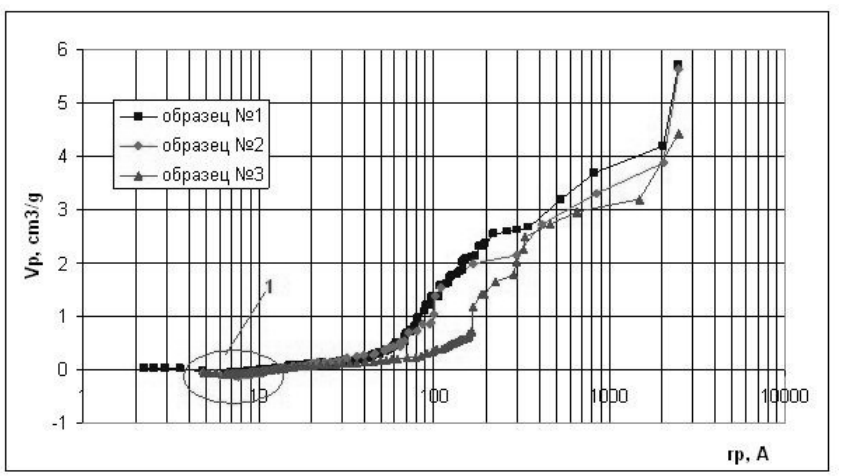

Fig. 6. Cumulative distribution curves in depending of pore radius. Sample №1 (source-propane-butane), №2 (methane), №3 (acetylene).

of slope of the pore size distribution for a sample with crumpled structure is greater. Structures differ by their pore volume. Main range of pore radii lies in the 10-70 $\AA$ range, corresponding to mesopores. The volume of pores with radius less than $70 \AA$ is around $0.8 \mathrm{~cm}^{3} / \mathrm{g}$.

A feature of all the graphene samples is the presence of an area 1 on a cumulative distribution curve (Fig. 6). The emergence of sub-zero values in this area was typical for the graphene samples obtained from previous studies. If you convert the schedule with respect to the pore radius by Kelvin (Fig. 6), excluding from the calculation standard thickness of the adsorption layer, resulting in the reference sample, it turns out the standard nature of the distribution curve. Perhaps this can be explained by the fact that graphene materials have different from other carbon materials structure of the adsorption film.

Detailed analysis of the samples shows that up to about $2 \mathrm{~nm}$ pore radius an adsorbed film thickness corresponds to the thickness of a monolayer or a little more. Up to this point adsorption curves have a customary character. When passing the border of less than $2 \mathrm{~nm}$, corresponding to the half-width of the adsorption film thickness of 1 to 1.5 monolayers, the character of the pores fill by the adsorption film changes abruptly compared to the reference material. This is due to the fact that the adsorption film thickness of the graphene is smaller than its thickness at graphitized soot (reference materials), that is included in the calculation. Obviously, the surface of a material more lyophobic with respect to benzene in comparison to the reference material.

\section{CONCLUSION}

Carried parametric study of the process of decomposition of hydrocarbons (a mixture of propane, butane, methane and acetylene) in the plasma jet generated by a DC plasma torch with divergent channel, showed that synthesis of nanotubes and graphene materials can be performed without the direct use of catalysts. Depending on the content of hydrogen in the hydrocarbon gases the nanostructures with different morphology (from disk up to crumpled) are formed. The flow rate of hydrocarbons and environment pressure are main parameters that influence the geometry of the structures. By the decomposition of a mixture of propane, butane optimal flow rate is $0.294 \mathrm{~m}^{3} / \mathrm{h}, 1.42 \mathrm{~m}^{3} / \mathrm{h}$ for methane, for acetylene is $0.202 \mathrm{~m}^{3} / \mathrm{h}$. Using propane - butane at environmental pressures of 350-500 Torr leads to the formation of graphene materials with a large average diameter size of $600 \mathrm{~nm}$, but using technical methane their size is increased up to $1 \mu \mathrm{m}$. The use of methane and acetylene in argon plasma provides the maximum output of crumpled structure (up to $95 \mathrm{wt} . \%$ ). In the first case these structures are formed at 650 Torr, while in the second case at 150 Torr. The resulting materials have a mesoporous structure that can serve as a template for the formation of supported catalyst systems.

The possibility of scaling graphene materials with desired characteristics are shown.

\section{ACKNOWLEDGEMENTS}

The work was supported by the Russian Science Foundation project №14-50-00124.

\section{REFERENCES}

1. Baimova UA, Murzaev RT, Dmitriev SV. Mechanical properties of bulk carbon nanomaterials. J. Physics of Solid State, 2014, 56(10):1947.

2. Shkolnikov EI, Witkina DE. Features nanoporous structure of carbon materials for supercapacitors. The limited vapor method. High Temperature, 2010, 48(6):854-861.

3. Peng C, Wen Z, Qin Y, Schmidt-Mende L, Li C, Yang S, Shi D, Yang J. Three-dimensional graphitized carbon nanovesicles for highperformance supercapacitors based on ionic liquids. ChemSusChem., 2014, 7(3):777- 
784.

4. Lim Siang Ying, Mohamad Amran bin Mohd Salleh, Hamdan b. Mohamed Yusoff, Suraya Binti Abdul Rashid, Jeefferie b. Abd. Razak Continuous production of carbon nanotubes. J. Industrial and Engineering Chem., 2011, 17(3):367. 